\title{
The Application of Runge-Kutta Schemes to Singular Initial Value Problems
}

\author{
By Frank de Hoog and Richard Weiss
}

\begin{abstract}
A theory for explicit Runge-Kutta schemes applied to the initial value problem for a first-order system of differential equations with a singularity of the first kind is developed. It is shown that, in general, the order of convergence is at most two but that the usual order up to a logarithmic term can be obtained for level three and four schemes applied to specific problems.
\end{abstract}

1. Introduction. In this paper we examine the application of explicit Runge-Kutta schemes to the numerical solution of the problem

$$
\begin{gathered}
y^{\prime}-M y / t=g(S y) / t+f(t, y), \quad 0<t \leqslant 1, \\
y \in C^{1}[0,1], \\
y(0)=\eta .
\end{gathered}
$$

Here $y, g, f$ are vector valued functions, $M, S$ are constant matrices, and $\eta$ is a vector. Further restrictions will be placed on $g, f, M, S$ and $\eta$ in the sequel.

Equations (1.1a, b) subject to the boundary condition $b(y(0), y(1))=0$ often arise when symmetry is used to reduce partial differential equations to ordinary differential equations. The interest in (1.1) is due to the fact that shooting techniques for the solution of the boundary value problem require the solution of the initial value problem.

When $M=0$ and $g=0$, explicit Runge-Kutta schemes are often used to calculate a numerical solution or provide starting values for multistep schemes, but the validity of this in the present case is questionable, since the equation has a singularity at $t=0$. However, in a number of numerical studies the application of explicit Runge-Kutta schemes to equations similar to (1.1) has yielded satisfactory starting values or provided an effective means of computing the solution on the entire interval, see Keller and Wolfe [4], Parter, Stein and Stein [6], and Rentrop [7]. This has motivated the examination presented here.

Although the order of convergence for the general case is shown to be poor, the usual order of convergence of level three and four schemes is established for a number of problems of practical interest.

Received August 1, 1983; revised May 3, 1984.,

1980 Mathematics Subject Classification. Primary 65L05.

Key words and phrases. Explicit Runge-Kutta schemes, initial value problem, singularity of the first kind, convergence. 
2. Analytic Background. Here we make a number of assumptions and present an existence and smoothness result for the solution of (1.1). It is assumed that

A2.1. The eigenvalues of $M$ have nonpositive real parts and the only possible eigenvalue on the imaginary axis is zero.

A2.2. $S$ is a projection of $\mathbf{R}^{n}$ onto the invariant subspace associated with the eigenvalues of $M$ that have negative real parts.

A2.3. $\eta \in \operatorname{ker} M$.

A2.4. The mappings $g, g_{y}, f$ and $f_{y}$ are well defined and continuous on $D_{g}$ and $D_{f}$, respectively, where $D_{g}$ is a convex open subset of $\mathbf{R}^{n}$ which contains $\eta$ and $D_{f}=[0,1] \times D_{g}$.

A2.5. There exists a constant $d$ such that

$$
\begin{array}{ll}
|g(y)| \leqslant d|y|^{2}, & y \in D_{g}, \\
\left|g_{y}(y)\right| \leqslant d|y|, & y \in D_{g} .
\end{array}
$$

Although these assumptions appear quite restrictive, they are often satisfied in practice. It is also frequently possible to bring singular systems into the form (1.1) by suitable transformations of the dependent variables (see Section 4).

In the sequel, $R$ denotes the spectral projection associated with the eigenspace of $M$ belonging to the zero eigenvalue, and $Q=I-R$.

The following theorem can be established by a combination of the techniques developed in [2] and contraction mapping arguments. (Compare also Theorem 2.1 in de Hoog and Weiss [3].)

THEOREM 2.1. There exists a constant $T>0$ such that (1.1) has a unique solution on $[0, T]$. This solution satisfies

$$
|Q y(t)| \leqslant \text { const } t \text {. }
$$

Furthermore, if $g$ and $f$ are $p$ times continuously differentiable on $D_{g}$ and $D_{f}$, respectively, then $y \in C^{p+1}[0,1]$.

3. Stability and Convergence. Explicit Runge-Kutta schemes for the solution of the initial value problem

$$
y^{\prime}(t)=F(t, y(t)), \quad 0 \leqslant t \leqslant T, y(0)=\eta,
$$

have the form

$$
\begin{aligned}
y_{j+1}-y_{j} & =h \sum_{r=1}^{m} w_{r} k_{r}\left(h, t_{j}, y_{j}\right) \\
& =h \Phi\left(h, t_{j}, y_{j}\right), \quad j=0, \ldots, N-1,
\end{aligned}
$$

where

$$
t_{0}=0, \quad t_{j+1}=t_{j}+h, \quad j=0, \ldots, N-1, \quad y_{0}=\eta,
$$

and

$$
k_{r}(h, t, y)=F\left(t+\alpha_{r} h, \quad y+h \sum_{q=1}^{r-1} \beta_{r q} k_{q}(h, t, y)\right), \quad r=1, \ldots, m
$$


In addition, we assume that

$$
\begin{aligned}
& \alpha_{1}=0, \quad \alpha_{r}=\sum_{q=1}^{r-1} \beta_{r q}>0, \\
& \sum_{r=1}^{m} w_{r}=1, \quad \sum_{r=2}^{m} w_{r} \alpha_{r}=1 / 2 .
\end{aligned}
$$

It is well known that the restrictions (3.2) ensure that the convergence of (3.1) is at least of order two for smooth $F(t, y)$.

For (1.1),

$$
F(t, y)=(M y+g(S y)) / t+f(t, y)
$$

and hence (3.1) is not well defined in the case when $j=0$, since an evaluation of $y^{\prime}(0)=F(0, \eta)$ is required. However,

$$
\lim _{t \rightarrow 0} \frac{g(S y(t))}{t}=0 \text { and } \lim _{t \rightarrow 0} \frac{M y(t)}{t}=M y^{\prime}(0),
$$

which yields

$$
y^{\prime}(0)=(I-M)^{-1} f(0, y(0)) .
$$

It is therefore appropriate to use

$$
F(0, y):=(I-M)^{-1} f(0, y) .
$$

Let

$H_{d, t_{j}}=\left\{x \in \mathbf{R}^{n}|| Q\left(x-y\left(t_{j}\right)\right)\left|\leqslant d t_{j},\right| R\left(x-y\left(t_{j}\right)\right) \mid \leqslant d\right\}, \quad j=0, \ldots, N-1$,

where $d$ is a positive constant. Then, for $d$ sufficiently small, it is easily shown that $\Phi\left(h, t_{j}, y_{j}\right)$ is well defined provided $y_{j} \in H_{d, t_{j}}$, and $h$ is small enough. Hence $y_{j+1}$ is well defined in this case and it follows from (3.1) that

$$
\varepsilon_{j+1}=\varepsilon_{j}+h \Phi\left(h, t_{j}, y_{j}\right)-h \Phi\left(h, t_{j}, y\left(t_{j}\right)\right)+\tau_{j}
$$

where $\varepsilon_{j}=y_{j}-y\left(t_{j}\right)$, and

$$
\tau_{j}=y\left(t_{j+1}\right)-y\left(t_{j}\right)-h \Phi\left(h, t_{j}, y\left(t_{j}\right)\right)
$$

is the local truncation error. Furthermore, it is not difficult to verify that for $y_{j} \in H_{d, t_{j}}$,

$$
h \Phi\left(h, t_{j}, y_{j}\right)-h \Phi\left(h, t_{j}, y\left(t_{j}\right)\right)=\left\{M /(j+1)+h A_{j}+B_{j} /(j+1)^{2}\right\} \varepsilon_{j},
$$

where

$$
\left|A_{j}\right|,\left|B_{j}\right| \leqslant \text { const, } \quad j=0, \ldots, N-1 .
$$

Substitution into $(3.4)$ yields

$$
\varepsilon_{j+1}=\left\{I+M /(j+1)+h A_{j}+B_{j} /(j+1)^{2}\right\} \varepsilon_{j}+\tau_{j}
$$

This equation is very similar to the difference equation studied in de Hoog and Weiss [2] for the stability of the centered Euler scheme and a straightforward 
modification of the analysis there yields

Lemma 3.1. Assume that (3.5) holds. Then, the difference equation

$$
\begin{aligned}
& u_{j+1}=\left\{I+M /(j+1)+h A_{j}+B_{j} /(j+1)^{2}\right\} u_{j} \\
&+h a_{j}+M b_{j} /(j+1)+c_{j} /(j+1)+e_{j} /(j+1)^{2}, \quad j=0, \ldots, N-1, \\
& u_{0}=0,
\end{aligned}
$$

has a unique solution. Furthermore,

$$
\left|u_{j}\right| \leqslant \operatorname{const}\left\{\log ^{p-1}(j+1)[b+e+c \log (j+1)]+a t_{j}\right\}, \quad j=1,2, \ldots, N,
$$

where

$$
a=\max _{j}\left|a_{j}\right|, \quad b=\max _{j}\left|M b_{j}\right|, \quad c=\max _{j}\left|c_{j}\right|, \quad e=\max _{j}\left|e_{j}\right|
$$

and $p$ is the smallest positive integer such that $\operatorname{rank}\left\{M^{p}\right\}=\operatorname{rank}\left\{M^{p+1}\right\}$.

We now estimate the local truncation error when $g$ and $f$ are three times continuously differentiable on $D_{g}$ and $D_{f}$, respectively. From Theorem 2.1, it then follows that $y \in C^{4}[0, T]$ for some $T>0$. Using Taylor's Theorem and (3.2), we find that

$$
\tau_{j}=h^{3} a_{j}+h^{2} M b_{j} /(j+1)+h^{2} e_{j} /(j+1)^{2}, \quad j=1, \ldots, N-1,
$$

where $\left|a_{j}\right|,\left|b_{j}\right|,\left|e_{j}\right| \leqslant$ const, $j=0, \ldots, N-1$. A simple inductive argument using (3.6), (3.7) and Lemma 3.1 now yields

THEOREM 3.1. The scheme (3.1) subject to (3.2) is well defined, provided $h$ is sufficiently small. Furthermore,

$$
\left|y_{j}-y\left(t_{j}\right)\right| \leqslant \text { const } h^{2} \log ^{p-1}(j+1), \quad j=1, \ldots, N .
$$

Runge-Kutta schemes, which are convergent of order $q \geqslant 2$ for equations without a singular point at $t=0$, have terms of the form $\partial F / \partial t, \partial^{2} F / \partial t^{2}$, etc., in the local truncation error. These terms yield

$$
\tau_{j}=O\left(h^{2} /(j+1)^{q-1}\right), \quad j=0, \ldots, N-1,
$$

when (3.1) is applied to (1.1). From Lemma 3.1 it therefore follows that in general the order of explicit Runge-Kutta schemes when applied to (1.1) is at most two.

However, we shall see in Section 4 that the usual order of convergence for level three $(m=3)$ and level four $(m=4)$ schemes may be obtained in particular problems. First we shall need sharper estimates of the local truncation error and these are given below.

Level Three Schemes $(m=3)$. In addition to (3.2), we assume that

$$
w_{2} \alpha_{2}^{2}+w_{3} \alpha_{3}^{2}=1 / 3, \quad w_{3} \alpha_{2} \beta_{32}=1 / 6
$$

These conditions lead to a two-parameter family of level three schemes that are convergent of order three when there is no singularity at $t=0$. (See, for example, Ralston [8, p. 199].) Using Taylor's Theorem, the local truncation error can be calculated to be

$$
\tau_{j}=-\frac{h^{4} \alpha_{2}\left(3 \alpha_{3}-2+M\right) M y^{\prime \prime}\left(t_{j}\right)}{12\left(t_{j}+\alpha_{2} h\right)\left(t_{j}+\alpha_{3} h\right)}+O\left(h^{3} /(j+1)\right), \quad j=0, \ldots, N-1 .
$$


Level Four Schemes $(m=4)$. In addition to (3.2) we assume that

$$
\begin{aligned}
& w_{2} \alpha_{2}^{2}+w_{3} \alpha_{3}^{2}+w_{4} \alpha_{4}^{2}=1 / 3 \\
& w_{2} \alpha_{2}^{3}+w_{3} \alpha_{3}^{3}+w_{4} \alpha_{4}^{3}=1 / 4, \\
& w_{3}^{2} \alpha_{2} \beta_{32}+w_{4}\left(\alpha_{2} \beta_{42}+\alpha_{3} \beta_{43}\right)=1 / 6, \\
& w_{3} \alpha_{2}^{2} \beta_{32}+w_{4}\left(\alpha_{2}^{2} \beta_{42}+\alpha_{3}^{2} \beta_{43}\right)=1 / 12, \\
& w_{3} \alpha_{2} \alpha_{3} \beta_{32}+w_{4}\left(\alpha_{2} \beta_{42}+\alpha_{3} \beta_{43}\right) \alpha_{4}=1 / 8, \\
& w_{4} \alpha_{2} \beta_{32} \beta_{43}=1 / 24
\end{aligned}
$$

Under suitable restrictions on $\alpha_{2}, \alpha_{3}$ (see Ralston [8, Problem 44, p. 225]) these conditions lead to a two-parameter family of level four schemes which are convergent of order four when there is no singular point at $t=0$. Note that one of the restrictions is $\alpha_{3} \neq 1$. A rather tedious but straightforward computation then yields

$$
\begin{aligned}
\tau_{j}= & -\frac{h^{5} \alpha_{2}\left(M+2\left(2 \alpha_{3}-1\right)\right)(M+1) M y^{\prime \prime}\left(t_{j}\right)}{48\left(t_{j}+\alpha_{2} h\right)\left(t_{j}+\alpha_{3} h\right)\left(t_{j}+h\right)} \\
& +\frac{h^{5}\left(4 \alpha_{2} \alpha_{3}+\alpha_{2}-2 \alpha_{3}\right)(M+1) M t_{j} y^{(3)}\left(t_{j}\right)}{144\left(t_{j}+\alpha_{2} h\right)\left(t_{j}+\alpha_{3} h\right)\left(t_{j}+h\right)} \\
& -\frac{h^{6} \alpha_{2}\left(\alpha_{2} M+2 \alpha_{3}-3 \alpha_{2}\right)(M+1) M y^{(3)}\left(t_{j}\right)}{144\left(t_{j}+\alpha_{2} h\right)\left(t_{j}+\alpha_{3} h\right)\left(t_{j}+h\right)} \\
& -\frac{h^{5} \alpha_{2}(M+1) M G\left(t_{j}\right) y^{\prime \prime}\left(t_{j}\right)}{48\left(t_{j}+\alpha_{3} h\right)\left(t_{j}+h\right)} \\
& -\frac{h^{5} \alpha_{2} G\left(t_{j}\right)\left(M+2\left(2 \alpha_{3}-1\right)\right) M y^{\prime \prime}\left(t_{j}\right)}{48\left(t_{j}+\alpha_{2} h\right)\left(t_{j}+\alpha_{3} h\right)} \\
& -\frac{h^{5} \alpha_{2} M G\left(t_{j}\right) M y^{\prime \prime}\left(t_{j}\right)}{48\left(t_{j}+\alpha_{3} h\right)\left(t_{j}+h\right)}+O\left(h^{4} /(j+1)\right),
\end{aligned}
$$

where

$$
G(t)=g_{y}(S y(t)) S / t+f_{y}(t, y(t))
$$

From A2.5 and Theorem 2.1, the matrix $G(t)$ is a smooth function of $t$.

\section{Applications of Runge-Kutta Schemes.}

\section{Example 1. Consider the problem}

$$
\Delta u=f(u)
$$

in a cylindrical or spherical domain. If we assume rotational symmetry, this leads to the boundary value problem,

$$
u^{\prime \prime}+\lambda u^{\prime} / t=f(u), \quad u^{\prime}(0)=0, \quad u(1)=\xi,
$$

where $\lambda=1$ or 2 , respectively. From symmetry, $u$ is an even function and Eq. (4.1) can be reduced to the form (1.1) in a number of ways. ( $u$ is said to be even if all odd derivatives vanish at zero.)

(a) Let $y_{1}=u, y_{2}=u^{\prime}$ and $y=\left(y_{1}, y_{2}\right)^{T}$. Then we obtain (1.1) with

$$
M=\left[\begin{array}{cc}
0 & 0 \\
0 & -\lambda
\end{array}\right], \quad g=0, \quad f(t, y)=\left(y_{2}, f\left(y_{1}\right)\right)^{T} .
$$


The relevant initial value when a shooting method is used is

$$
\eta=\left(\eta_{1}, 0\right)^{T}
$$

Clearly, $(M+\lambda) M=0$, and since $u$ is even,

$$
M y^{\prime \prime}(t)=\left(0,-\lambda u^{(3)}(t)\right)^{T}=O(t) .
$$

Hence, for $m=3$ :

$$
\tau_{j}=O\left(h^{3} /(j+1)\right)
$$

Whence

$$
\left|y_{j}-y\left(t_{j}\right)\right| \leqslant \text { const } h^{3} \log (j+1),
$$

and for $m=4, \lambda=1$ :

$$
\tau_{j}=O\left(h^{4} /(j+1)\right)
$$

and so

$$
\left|y_{j}-y\left(t_{j}\right)\right| \leqslant \text { const } h^{4} \log (j+1)
$$

For $m=4, \lambda=2$ :

$$
\begin{aligned}
\tau_{j}= & \frac{h^{5}\left(3 \alpha_{2} M+8 \alpha_{2} \alpha_{3}-7 \alpha_{2}+2 \alpha_{3}\right) M t_{j} y^{(3)}\left(t_{j}\right)}{144\left(t_{j}+\alpha_{2} h\right)\left(t_{j}+\alpha_{3} h\right)\left(t_{j}+h\right)} \\
& +\frac{h^{6} \alpha_{2}\left(\alpha_{2} M+2 \alpha_{3}-3 \alpha_{2}\right) M y^{(3)}\left(t_{j}\right)}{144\left(t_{j}+\alpha_{2} h\right)\left(t_{j}+\alpha_{3} h\right)\left(t_{j}+h\right)} \\
& +\frac{h^{5} \alpha_{2} M G\left(t_{j}\right) y^{\prime \prime}\left(t_{j}\right)}{48\left(t_{j}+\alpha_{3} h\right)\left(t_{j}+h\right)}+O\left(h^{4} /(j+1)\right) \\
= & O\left(h^{3} /(j+1)^{2}\right) .
\end{aligned}
$$

In this case we cannot choose $\alpha_{2}$ and $\alpha_{3}$ to further increase the order of the truncation error. The numerical solution will therefore satisfy

$$
\left|y_{j}-y\left(t_{j}\right)\right| \leqslant \text { const } h^{3} \text {. }
$$

(b) Let $y_{1}=u, y_{2}=t u^{\prime}$ and $y=\left(y_{1}, y_{2}\right)^{T}$. Then we obtain (1.1) with

$$
M=\left[\begin{array}{cc}
0 & 1 \\
0 & 1-\lambda
\end{array}\right], \quad g=0, \quad f(t, y)=\left(0, t f\left(y_{1}\right)\right)^{T} .
$$

Clearly, $y$ is even and

$$
G(t)=O(t), \quad M(M+\lambda-1)=0 .
$$

Hence, for $m=3$ :

$$
\tau_{j}=-\frac{h^{4} \alpha_{2}\left(3 \alpha_{2}-1-\lambda\right) M y^{\prime \prime}\left(t_{j}\right)}{12\left(t_{j}+\alpha_{2} h\right)\left(t_{j}+\alpha_{3} h\right)}+O\left(h^{3} /(j+1)\right),
$$

and, in general, we have

$$
\left|y_{j}-y\left(t_{j}\right)\right| \leqslant \text { const } h^{2} \text {. }
$$


However, by choosing $\alpha_{3}=(1+\lambda) / 3$, the first term in the truncation error is zero and in this case we find that

$$
\left|y_{j}-y\left(t_{j}\right)\right| \leqslant \text { const } h^{3}(\log (j+1))^{3-\lambda} \text {. }
$$

For $m=4, \lambda=1$ :

$$
\tau_{j}=-\frac{h^{5} \alpha_{2}\left(2 \alpha_{3}-1\right) M y^{\prime \prime}\left(t_{j}\right)}{24\left(t_{j}+\alpha_{2} h\right)\left(t_{j}+\alpha_{3} h\right)\left(t_{j}+h\right)}+O\left(h^{4} /(j+1)\right),
$$

and so

$$
\left|y_{j}-y\left(t_{j}\right)\right| \leqslant \text { const } h^{2} .
$$

However, if we take $\alpha_{3}=1 / 2$, then the leading term in the truncation error is zero and therefore

$$
\left|y_{j}-y\left(t_{j}\right)\right| \leqslant \text { const } h^{4}(\log (j+1))^{2} \text {. }
$$

For $m=4, \lambda=2$ :

$$
\tau_{j}=O\left(h^{4} /(j+1)\right)
$$

and hence

$$
\left|y_{j}-y\left(t_{j}\right)\right| \leqslant \text { const } h^{4} \log (j+1) .
$$

Example 2. Keller and Wolfe [4] consider the system

$$
\mathscr{L} u(t)=\rho\left[u(t) v(t)+P t^{2}\right], \quad \mathscr{L} v(t)=\rho\left[t^{2}-u^{2}(t)\right]
$$

subject to

$$
u(0)=v(0)=0 ; \quad u(1)=1, \quad v^{\prime}(1)=\nu v(1),
$$

where $\mathscr{L}$ is the differential operator

$$
\mathscr{L} \cdot=t \frac{d}{d t}\left(\frac{1}{t} \frac{d}{d t}(t \cdot)\right)
$$

and $\rho, P, \nu$ are constants. This problem arises in the study of the buckling mechanism of the cap portion of a spherical shell. From symmetry, $u$ and $v$ are odd functions.

(a) Let

$$
\begin{gathered}
y_{1}(t)=u(t), \quad y_{2}(t)=\frac{1}{t} \frac{d}{d t}(t u(t)), \\
y_{3}(t)=v(t), \quad y_{4}(t)=\frac{1}{t} \frac{d}{d t}(t v(t)), \\
y=\left(y_{1}, y_{2}, y_{3}, y_{4}\right)^{T} .
\end{gathered}
$$

Then we obtain (1.1) with

$$
\begin{gathered}
M=\left[\begin{array}{cccc}
-1 & 0 & 0 & 0 \\
0 & 0 & 0 & 0 \\
0 & 0 & -1 & 0 \\
0 & 0 & 0 & 0
\end{array}\right], \\
g(S y)=\left(0, \rho y_{1} y_{3}, 0,-\rho y_{1}^{2}\right)^{T} \text { and } f(t, y)=\left(y_{2}, \rho P t, y_{4}, \rho t\right)^{T} .
\end{gathered}
$$


Furthermore, it can easily be verified that $M y$ is odd, $M G(t) M=0$ and $M(M+1)$ $=0$. Hence

For $m=3$ :

$$
\tau_{j}=O\left(h^{3} /(j+1)\right) .
$$

The global discretization error is therefore $O\left(h^{3} \log (j+1)\right)$.

For $m=4$ :

$$
\tau_{j}=O\left(h^{4} /(j+1)\right) .
$$

Thus, the global discretization error is $O\left(h^{4} \log (j+1)\right)$.

(b) Let

$$
\begin{array}{cl}
y_{1}(t)=u(t) / t, & y_{2}(t)=u^{\prime}(t), \\
y_{3}(t)=v(t) / t, & y_{4}(t)=v^{\prime}(t), \\
y=\left(y_{1}, y_{2}, y_{3}, y_{4}\right)^{T} .
\end{array}
$$

Then we obtain (1.1) with

$$
\begin{gathered}
M=\left[\begin{array}{cccc}
-1 & 1 & 0 & 0 \\
1 & -1 & 0 & 0 \\
0 & 0 & -1 & 1 \\
0 & 0 & 1 & -1
\end{array}\right], \\
g(S y)=0
\end{gathered}
$$

and

$$
f(t, y)=\left(0, t \rho\left(y_{1} y_{3}+P\right), 0, t \rho\left(1-y_{1}^{2}\right)\right)^{T} .
$$

Appropriate initial conditions are $y(0)=\left(\eta_{1}, \eta_{3}, \eta_{3}, \eta_{3}\right)^{T}$ and it is easy to verify that $y$ is an even function. Furthermore, $G(t)=O(t)$ and $M(M+2)=0$.

Hence, for $m=3$ :

$$
\tau_{j}=\frac{h^{4} \alpha_{2}\left(4-3 \alpha_{3}\right) M y^{\prime \prime}\left(t_{j}\right)}{12\left(t_{j}+\alpha_{2} h\right)\left(t_{j}+\alpha_{3} h\right)}+O\left(h^{3} /(j+1)\right) .
$$

The global discretization error is therefore $O\left(h^{2}\right)$ unless $\alpha_{3}=4 / 3$ in which case it is $O\left(h^{3} \log (j+1)\right)$.

For $m=4$ :

$$
\tau_{j}=\frac{-h^{5} \alpha_{2}\left(1-\alpha_{3}\right) M y^{\prime \prime}\left(t_{j}\right)}{12\left(t_{j}+\alpha_{2} h\right)\left(t_{j}+\alpha_{3} h\right)\left(t_{j}+h\right)}+O\left(h^{4} /(j+1)\right)
$$

and hence, the global discretization error is $O\left(h^{2}\right)$. Note that it is not possible to take $\alpha_{3}=1$ since, in this case, there does not exist a level four scheme which is convergent of order four when there is no singularity at $t=0$. The form of the local discretization error does, however, suggest the following change of variables:

(c)

$$
\begin{gathered}
y_{1}(t)=u(t) / t-\gamma t^{2}, \quad y_{2}(t)=u^{\prime}(t)-3 \gamma t^{2}, \\
y_{3}(t)=v(t) / t-\sigma t^{2}, \quad y_{4}(t)=v^{\prime}(t)-3 \sigma t^{2}, \\
y=\left(y_{1}, y_{2}, y_{3}, y_{4}\right)^{T}
\end{gathered}
$$


where

$$
\gamma=\rho\left[\eta_{1} \eta_{3}+P\right] / 8, \quad \sigma=\rho\left[1-\eta_{1}^{2}\right] / 8,
$$

and

$$
\eta_{1}=y_{1}(0)=y_{2}(0), \quad \eta_{3}=y_{3}(0)=y_{4}(0) .
$$

This leads to an equation of the form (1.1) where $M$ and $g$ are given by (4.10) and (4.11), respectively, and

$$
\begin{aligned}
& f(t, y)=\left(0,-8 \gamma t+t \rho\left[\left(y_{1}+\gamma t^{2}\right)\left(y_{3}+\sigma t^{2}\right)+P\right]\right. \\
&\left.0,-8 \sigma t+t \rho\left[1-\left(y_{1}+\gamma t^{2}\right)\right]\right)^{T} .
\end{aligned}
$$

It is not difficult to verify that $y^{\prime \prime}(t)=O\left(t^{2}\right)$ and, hence, that for level three and four schemes the global discretization error decreases like $\left(h^{3} \log (j+1)\right)$ and $\left(h^{4} \log (j+1)\right)$, respectively.

A similar analysis can be applied to other problems of practical interest such as the shell equations derived in Bauer, Reiss and Keller [1] and the Ginzburg-Landau equations discussed in Meissner and Tholfsen [5].

5. Numerical Results. In this section we illustrate the theory developed in the previous sections by some numerical examples. Specifically, we consider the schemes defined by the parameters

$$
\begin{aligned}
& \beta_{21}=1 / 2, \quad \beta_{31}=0, \quad \beta_{32}=3 / 4, \\
& \alpha_{1}=0, \quad \alpha_{2}=1 / 2, \quad \alpha_{3}=3 / 4, \\
& w_{1}=2 / 9, \quad w_{2}=1 / 3, \quad w_{3}=4 / 9 \text {. } \\
& \beta_{21}=1 / 3, \quad \beta_{31}=0, \quad \beta_{32}=2 / 3 \text {, } \\
& \alpha_{1}=0, \quad \alpha_{2}=1 / 3, \quad \alpha_{3}=2 / 3, \\
& w_{1}=1 / 4, \quad w_{2}=0, \quad w_{3}=3 / 4 \text {. } \\
& \beta_{21}=1 / 2, \quad \beta_{31}=0, \quad \beta_{32}=1 / 2 \text {, } \\
& \beta_{41}=0, \quad \beta_{42}=0, \quad \beta_{43}=1 \text {, } \\
& \alpha_{1}=0, \quad \alpha_{2}=1 / 2, \quad \alpha_{3}=1 / 2, \\
& \alpha_{4}=1, \quad w_{1}=1 / 6, \quad w_{2}=1 / 3, \\
& w_{3}=1 / 3, \quad w_{4}=1 / 6 \text {. } \\
& \beta_{21}=1 / 3, \quad \beta_{31}=-1 / 3, \quad \beta_{32}=1 \text {, } \\
& \beta_{41}=1, \quad \beta_{42}=-1, \quad \beta_{43}=1 \text {, } \\
& \alpha_{1}=0, \quad \alpha_{2}=1 / 3, \quad \alpha_{3}=2 / 3, \\
& \alpha_{4}=1, \quad w_{1}=1 / 8, \quad w_{2}=3 / 8, \\
& w_{3}=3 / 8, \quad w_{4}=1 / 8 \text {. }
\end{aligned}
$$

Clearly, (5.1) and (5.2) are level three schemes while (5.3) and (5.4) are level four schemes. The parameters have been chosen so that the level three schemes are convergent of order three, and the level four schemes are convergent of order four in the absence of a singular point.

In Table 5.1, we have tabulated the maximum error when schemes (5.1)-(5.4) are applied to problem (4.2) with $\lambda=1, f(u)=u^{3}-3 u^{5}$ and $\eta_{1}=1$. The solution is 
$y_{1}(t)=\left(1+t^{2}\right)^{-1 / 2}, y_{2}(t)=-t\left(1+t^{2}\right)^{-3 / 2}$. The error estimate (4.3) for schemes (5.1), (5.2) and the estimate (4.4) for schemes (5.3), (5.4) are borne out by this example. Thus, for this problem, we have the usual order of convergence up to logarithmic terms.

TABLE 5.1.

Maximum errors for problem (4.2)

\begin{tabular}{|l|c|c|c|c|}
\hline \multicolumn{1}{|c|}{$h$} & $\begin{array}{c}\text { scheme } \\
(5.1)\end{array}$ & $\begin{array}{c}\text { scheme } \\
(5.2)\end{array}$ & $\begin{array}{c}\text { scheme } \\
(5.3)\end{array}$ & $\begin{array}{c}\text { scheme } \\
(5.4)\end{array}$ \\
\hline $1 / 4$ & $.791 \cdot 10^{-2}$ & $.856 \cdot 10^{-2}$ & $.106 \cdot 10^{-2}$ & $.104 \cdot 10^{-2}$ \\
$1 / 8$ & $.982 \cdot 10^{-3}$ & $.100 \cdot 10^{-2}$ & $.840 \cdot 10^{-4}$ & $.894 \cdot 10^{-4}$ \\
$1 / 16$ & $.122 \cdot 10^{-3}$ & $.123 \cdot 10^{-3}$ & $.576 \cdot 10^{-5}$ & $.687 \cdot 10^{-5}$ \\
$1 / 32$ & $.153 \cdot 10^{-4}$ & $.153 \cdot 10^{-4}$ & $.372 \cdot 10^{-6}$ & $.505 \cdot 10^{-6}$ \\
$1 / 64$ & $.191 \cdot 10^{-5}$ & $.191 \cdot 10^{-5}$ & $.236 \cdot 10^{-7}$ & $.362 \cdot 10^{-7}$ \\
\hline
\end{tabular}

We now present some results which demonstrate that the usual order of convergence is not necessarily achieved for equations with a singular point. From any fixed point $\bar{t}$ onwards, the numerical schemes will, of course, retain their usual order of convergence. However, due to the potential reduction of accuracy in the numerical solution on $[0, \bar{t}]$, the numerical initial values at $\bar{t}$ have errors of the orders discussed in the preceding section. These errors dominate the errors accumulated in the remaining computation and we have therefore chosen to present the numerical results by tabulating the error in a fixed component at the point $\bar{t}=1 / 4$.

Table 5.2 tabulates the error in the second component of the solution at $\bar{t}=1 / 4$ when the schemes are applied to problem (4.5) with $\lambda, \eta$, and $f$ as previously. The solution is now $y_{1}(t)=\left(1+t^{2}\right)^{-1 / 2}, y_{2}(t)=-t^{2}\left(1+t^{2}\right)^{-3 / 2}$. For scheme (5.1) the estimate (4.6) is appropriate but scheme (5.2) has $\alpha_{3}=2 / 3$ and hence the bound given by (4.7) is appropriate. Similarly, scheme (5.4) satisfies (4.8) but scheme (5.3) has $\alpha_{3}=1 / 2$ and thus the error satisfies (4.9). Again the numerical results show agreement with the theory and demonstrate that schemes applied to problems with a singular point do not necessarily yield the usual order of convergence.

TABLE 5.2

$$
y_{2, N / 4}-y_{2}(1 / 4) \text { for problem }(4.5)
$$

\begin{tabular}{|l|c|c|c|c|}
\hline \multicolumn{1}{|c|}{$h$} & $\begin{array}{c}\text { scheme } \\
(5.1)\end{array}$ & $\begin{array}{c}\text { scheme } \\
(5.2)\end{array}$ & $\begin{array}{c}\text { scheme } \\
(5.3)\end{array}$ & $\begin{array}{c}\text { scheme } \\
(5.4)\end{array}$ \\
\hline $1 / 4$ & $-.543 \cdot 10^{-2}$ & $-.543 \cdot 10^{-2}$ & $.135 \cdot 10^{-2}$ & $-.344 \cdot 10^{-3}$ \\
$1 / 8$ & $-.724 \cdot 10^{-3}$ & $-.465 \cdot 10^{-3}$ & $.147 \cdot 10^{-3}$ & $-.139 \cdot 10^{-3}$ \\
$1 / 16$ & $-.125 \cdot 10^{-3}$ & $-.406 \cdot 10^{-4}$ & $.112 \cdot 10^{-4}$ & $-.490 \cdot 10^{-4}$ \\
$1 / 32$ & $-.261 \cdot 10^{-4}$ & $-.382 \cdot 10^{-5}$ & $.772 \cdot 10^{-6}$ & $-.135 \cdot 10^{-4}$ \\
$1 / 64$ & $-.605 \cdot 10^{-5}$ & $-.391 \cdot 10^{-6}$ & $.498 \cdot 10^{-7}$ & $-.346 \cdot 10^{-5}$ \\
\hline
\end{tabular}


6. Concluding Remarks. Although, in general, the order of convergence of explicit Runge-Kutta schemes applied to equations of the form (1.1) is at most two, higher-order convergence can be obtained for particular problems. However, each problem requires individual analysis and it may be necessary to transform the problem to a more appropriate form before applying a scheme. In addition, the scheme used should depend on the problem being solved.

Finally, it should be noted that the theory developed can easily be extended to quasi-uniform grids.

Division of Mathematics and Statistics

CSIRO

P. O. Box 1965

Canberra, Australia

Institut für Angewandte und Numerische Mathematik

Technische Universitaet

Gusshausstrasse 27-29

A-1040 Wien, Austria

1. L. BAuer, E. L. Reiss \& H. B. Keller, “Axisymmetric buckling of hollow spheres and hemispheres," Comm. Pure Appl. Math., v. 23, 1970, pp. 529-568.

2. F. DE HOOG \& R. WEISs, "Difference methods for boundary value problems with a singularity of the first kind," SIAM J. Numer. Anal., v. 13, 1976, pp. 775-813.

3. F. DE HOOG \& R. WEISs, "The application of linear multistep methods to singular initial value problems," Math. Comp., v. 31, 1977, pp. 676-690.

4. H. B. Keller \& A. W. Wolfe, "On the non-unique equilibrium states and buckling mechanism of spherical shells,” J. Soc. Indust. Appl. Math., v. 13, 1965, pp. 674-705.

5. H. MeisSNer \& P. ThOlfSEN, "Cylindrically symmetric solutions of the Ginzburg-Landau equations," Phys. Rev., v. 169, 1968, pp. 413-416.

6. S. V. PARTer, M. L. Stein \& P. R. Stein, On the Multiplicity of Solutions of a Differential Equation Arising in Chemical Reactor Theory, Computer Sciences Technical Report \#194, University of Wisconsin-Madison, 1973.

7. P. RENTROP, "A Taylor series method for the numerical solution of two point boundary value problems," Numer. Math., v. 31, 1979, pp. 359-375.

8. A. Ralston, A First Course in Numerical Analysis, McGraw-Hill, New York, 1965. 